\title{
Comportamento de consumo de alimentos de famílias de baixa renda de pequenas cidades brasileiras: o caso de Mato Grosso do Sul
}

\author{
Food consumption behavior of low-income households from families of small \\ brazilians cities: the case of Mato Grosso do Sul
}

\section{Comportement de la consommation alimentaire des familles à faible revenu dans les petites villes brésilien: le cas du Mato Grosso do Sul \\ Comportamiento del consumo de alimentos de las familias de bajos ingresos de pequeños ciudades de Brasil: el caso en Mato Grosso do Sul}

\author{
Lilliane Renata Defante* \\ (lillianerenata@gmail.com) \\ Leidy Diana Oliveira Nascimento** \\ (leidynha_diana@yahoo.com.br) \\ Dario de Oliveira Lima-Filho* \\ (dariolimafilho@gmail.com)
}

Recebido em 18/09/2014; revisado e aprovado em 24/10/2014; aceito em 22/11/2014

DOI: http://dx.doi.org/10.1590/151870122015203

\begin{abstract}
Resumo: Este trabalho teve como objetivo analisar os hábitos alimentares de famílias de baixa renda nos territórios dos Consórcios de Segurança Alimentar e Desenvolvimento Local de Mato Grosso do Sul (CONSAD). Foi feito um estudo quantitativo-descritivo junto a 584 indivíduos moradores dos 24 municípios abrangidos, pertencentes às classes sociais C2, D e E, no ano de 2010, utilizando questionário estruturado. Os resultados mostram a existência de dois clusters, nomeados de segmento "saudável" e segmento "apreciadores de comida".

Palavras-chave: Alimentação. Comportamento alimentar. Cultura alimentar.

Abstract: This study aimed to examine the eating habits of low-income families in the territories of the Consortium for Food Security and Local Development of Mato Grosso do Sul (CONSAD). A quantitative-descriptive study was done with the 584 individuals living in the territory of the municipalities of CONSAD, social classes C2, D and E, in 2010, using a structured questionnaire. The results showed the existence of two clusters, named "healthy" segment and segment "food lovers".

Key words: Food. Feeding behavior. Food culture.

Résumé: Cette étude visait à examiner les habitudes alimentaires des familles à faible revenu dans les territoires du Consortium pour la sécurité alimentaire et le développement local du Mato Grosso do Sul (CONSAD). Une étude quantitative descriptive a été réalisée avec les 584 personnes qui vivent sur le territoire des municipalités de CONSAD, classes sociales C2, D et E, en 2010, à l'aide d'un questionnaire structuré. Les résultats ont montré l'existence de deux groupes, nommés secteur «santé» et «amoureux de la nourriture" segment.

Mots-clés: Alimentation. Comportement alimentaire. De la culture alimentaire.

Resúmen: Este estudio tuvo como objetivo examinar los hábitos alimenticios de las familias de bajos ingresos en los territorios del Consorcio de Seguridad Alimentaria y Desarrollo Local de Mato Grosso do Sul (CONSAD). Un estudio cuantitativo descriptivo se hizo con las 584 personas que viven en el territorio de los municipios de CONSAD, clases sociales C2, D y E, en 2010, utilizando un cuestionario estructurado. Los resultados mostraron la existencia de dos grupos, denominados segmento "saludable" y "amantes de la comida" del segmento.

Palabras clave: Alimento. Comportamiento de alimentación. La cultura alimentaria.
\end{abstract}

\section{Introdução}

A escolha alimentar é despertada muito cedo, desde a infância, por meio das sensações (tátil, gustativa, olfativa) sobre o que se come, e é pouco permeável homogeneizar essas escolhas de acordo com a imposição feita pela produção e distribuição massificada. A escolha alimentar também faz uma interlocução com os recursos, gostos e hábitos, e dependem de maneira direta da classe social do indivíduo e da cultura em que vive (CANESQUI; GARCIA, 2005). O estudo dos hábitos alimentares tem papel fundamental para identificar o que os consumidores adquirem em termos de alimentos e, também, quais os fatores que permeiam a escolha deles.

\footnotetext{
* Universidade Federal de Mato Grosso do Sul (UFMS), Campo Grande, Mato Grosso do Sul, Brasil.

** Ministério da Ciência, Tecnologia e Inovação (MCT\&I), Brasília, Distrito Federal, Brasil.
} 
O consumo de alimentos está aumentando globalmente, porém os padrões de consumo variam de acordo com os países e os níveis de renda (LIMA-FILHO; OLIVEIRA, 2009; REGMI; GEHLHAR, 2005). Estudos prévios (FAO, 2008; INGLIS et al., 2009; MISHRA et al., 2002) mostram que a renda é um fator determinante do comportamento de consumo de alimentos, embora existam outras variáveis relevantes.

Para definir os hábitos, a cultura e o comportamento alimentar da população de baixa renda, é preciso atentar-se à definição dessa classe social. De acordo com o IBGE (2006), famílias de baixa renda são aquelas com poder de compra até 2 salários mínimos, consideradas de nível E (classe baixa); entre 2 e 5 salários mínimos, denominada classe D; e as famílias de 4 a 8 salários mínimo são consideradas C2 (renda média baixa). A mesma fonte revela que todas essas três classes juntas representam $45 \%$ dos lares urbanos brasileiros; esse dado mostra que o poder individual dessas classes é baixo, dispondo de apenas $\mathrm{R} \$$ 35,00 diários para gastar com alimentação.

Por volta de três quartos das famílias brasileiras pertencem hoje às classes $C, D$ e E, conforme o Critério Econômico Brasil (IBGE, 2006). Essas famílias respondem por $42 \%$ do consumo do país e mobilizam, por ano, cerca de R\$390 bilhões (CHAUVEL, MATTOS, 2008).

A pesquisa do orçamento familiar [POF 2008-2009] (IBGE, 2010) avaliou a quantidade consumida de alimentos pelas famílias diante de três possibilidades de resposta: normalmente insuficiente; às vezes insuficiente; e sempre suficiente. Esses dados confrontados com o da POF de 2003 apresentaram robusta melhora da satisfação das famílias em todas as Regiões do Brasil. Mais de $60 \%$ das famílias $(64,5 \%)$ avaliaram como suficiente a quantidade de alimento, ante $53 \%$ da pesquisa de 2003. Em contrapartida, $35 \%$ das famílias diziam que a quantidade de alimento consumido normalmente ou eventualmente era insuficiente, dado que confronta os quase $47 \%$ da pesquisa anterior. Os estados que apresentaram maior satisfação na quantidade de alimentos consumidos foram Rio Grande do Sul e Santa Catarina (80,7\% sempre suficiente). Mato Grosso do Sul (MS), na Região Centro-Oeste, apresentou $77 \%$ das famílias com resposta para sempre suficientes.
Entre as famílias (no Brasil) que avaliaram como insuficiente, 20,3\% registravam até $\mathrm{R} \$ 1.245,00$ de rendimento familiar; $14,5 \%$ recebiam de $\mathrm{R} \$ 1.245,00$ a $\mathrm{R} \$ 2.490,00$; cerca de $10 \%$ têm rendimento de $\mathrm{R} \$ 2.049,00$ a $\mathrm{R} \$ 6.225,00$; e $2,4 \%$ recebiam acima de $\mathrm{R} \$$ 6.225,00 mensalmente. Os rendimentos versus gasto mensal com alimentos são especificados pelo IBGE (2010), segundo o qual famílias que têm rendimentos de até $R \$ 830,00$ mensais gastam $\mathrm{R} \$ 207,15$ com alimentação; as famílias com rendimento mensal de $\mathrm{R} \$ 830,00$ a $R \$ 1.245,00$ gastam $R \$ 279,02$ com alimentação mensalmente; famílias com rendimento mensal de $\mathrm{R} \$ 1.245,00$ a $\mathrm{R} \$ 2.490,00$ gastam $\mathrm{R}$ \$ 378,83 mensais com alimentação; as famílias com rendimento mensal de $\mathrm{R} \$ 2.490,00 \mathrm{a}$ $\mathrm{R} \$$ 4.150,00 gastam $\mathrm{R} \$ 522,66$ mensalmente com alimentação.

$O$ estudo da renda familiar não abrange $100 \%$ das famílias, já que a POF só inclui famílias com domicílios temporários ou permanentes, ficando de fora aqueles que não possuem moradia. Não se incluem na renda os recursos de doações ou de programas do governo nem a produção para autoconsumo, as quais podem ter um impacto elevado no consumo alimentar.

O hábito alimentar da população brasileira originou-se a partir da miscigenação de culturas existentes ou trazidas ao país. No Brasil, a alimentação teve influência da cultura indígena, por meio do modo de economia alimentar que eles utilizavam, como a atividade coletora, vivendo à custa da natureza e utilizando a mandioca na produção de variados alimentos. A alimentação vegetariana teve papel fundamental na atividade de subsistência, bem como a caça e a pesca. A contribuição da cultura alimentar de Portugal era mostrar a existência da oliva; já os africanos introduziram o consumo de manga, de arroz e da cana-de-açúcar. Na atualidade, foram percebidas importantes mudanças nos hábitos alimentares dos brasileiros, tais como: a redução no consumo per capita de arroz, o aumento do consumo da carne, ovos e laticínios, e um incremento nos gastos com produtos industrializados (CONTRERAS; GRACIA, 2011).

Mas nem toda a população pode usufruir de alimentos saudáveis e em quantidades suficientes regularmente. Concernente a 
essa afirmação, Monteiro (2003) afirma que a população de baixa renda, mesmo tendo acesso ao alimento, se mostra propensa a economizar na compra, o que pode significar a redução da qualidade de vida e da variedade. Segundo Belik (2003), a fome não está apenas ligada à quantidade de alimentos consumidos, mas, também, à qualidade desses alimentos. Assim, um estudo para verificar os hábitos alimentares de uma determinada população proporciona base para a formulação de políticas públicas de Segurança Alimentar e Nutricional (SAN).

A SAN significa dar garantia e condições de acesso a alimentos básicos de qualidade a todos, em quantidade suficiente, de modo permanente e sem comprometer o acesso a outras necessidades essenciais, com base em práticas alimentares saudáveis, contribuindo, assim, para uma existência digna, considerando todos os aspectos que são importantes para o desenvolvimento completo de um indivíduo saudável (OMS, 2003). Assim, para a melhor compreensão da SAN, existem quatro estágios como sugeridos por Weingartner (2004):

i) "disponibilidade" refere-se à existência física do alimento na produção ou no próprio mercado;

ii) "acesso" é garantido quando toda a família, todos os indivíduos dentro deste agregado familiar possuem recursos suficientes para obter uma alimentação apropriada para uma dieta nutritiva, mas o acesso ao alimento depende, ainda, do ambiente físico, político e social;

iii) "consumo de alimentos" refere-se ao aspecto socioeconômico da segurança alimentar da família, se o alimento é suficiente e nutritivo ou disponível e acessível;

iv) "absorção biológica de alimentos" significa que a pessoa deve ter uma boa saúde para assimilar os alimentos ingeridos.

Neste estudo, analisou-se a etapa "consumo de alimentos". O consumo é dependente da cultura alimentar de uma determinada população. Especificamente neste trabalho, os hábitos alimentares da população de baixa renda são considerados proxies da cultura alimentar.

De acordo com Poulain (2004), pesquisas científicas atreladas ao conhecimento científico podem ser usadas para aprimorar os padrões de alimentação da população, no que diz respeito a técnicas de produção, com um "olhar" mais crítico aos riscos sanitários, sem interferir nas dimensões gustativas e/ ou simbólicas, assim como podem ajudar no reconhecimento da população de risco conduzindo de melhor forma as políticas informacionais.

Em nível microeconômico, os critérios de consumo são usados para avaliar as implicações de preço versus renda sobre o estado nutricional, particularmente entre as pessoas com menos renda. São importantes, também, para idealizar ou melhorar programas governamentais que representam a população, no âmbito da identificação de grupos vulneráveis à insegurança alimentar e nutricional para que logo sejam desenvolvidas políticas públicas a esses grupos, de acordo com sua colocação social, atendendo, assim, às necessidades específicas que foram identificadas.

Dessa forma, no que diz respeito a políticas públicas, este trabalho contribui como embasamento de inclusão social que surge a partir da variável consumo. Tratando-se de políticas agrícolas e alimentares, ao aperfeiçoarem as condições de saúde da população e darem auxílio a outras vertentes, como a fome, a desnutrição e a mortalidade infantil, podem proporcionar emprego e renda à população, exercendo efeito proliferador da economia e, assim, contribuindo diretamente no desenvolvimento econômico, local e social de MS.

O trabalho traz contribuição gerencial, pois, como se trata da análise do comportamento de consumo de uma determinada população residente em pequenas cidades de MS, oferece uma base para desenvolver estratégias que sejam adequadas a esse mercado. À luz da academia, o estudo pode contribuir com uma discussão sobre a caracterização do comportamento do consumidor de baixa renda no interior do Brasil, permitindo uma compreensão mais apurada das suas determinantes.

Muitos estudos realizados para medir o comportamento de consumo de alimentos focam no viés econômico, como renda e preço (CUDJE; BREISINGER; DIAO, 2010; POWELL; ZHAO; WANG, 2009; MYERS, 2006) e, mesmo que prevejam as preferências individuais, não as investigam profundamente. Já os estudos do comportamento do consumidor de alimentos atrelados ao marketing costumam focar no hábito e preferência da população, com o 
intuito de estratificar o mercado e determinar públicos-alvo (LENGARD; JOHANSEN; HERSLETH, 2010; LEIBTAG; KAUFMAN, 2003; BRADY; ROBERTSON; CRONIN, 2001). Entender quais são os hábitos ou comportamentos alimentares de uma população torna-se importante quando se fala de baixa renda. Cada vez mais, o problema alimentar é considerado de ordem mundial, no qual os modelos de consumo são representados por desigualdade, da insuficiência ao desperdício, o que acarreta desequilíbrios nutricionais.

Assim, entender os hábitos e preferências alimentares dos consumidores ou da população significa compreender como eles estão dispostos a gastar os recursos (dinheiro, tempo e esforço). No entanto são diversos os fatores que influenciam esse processo de tomada de decisão (BELIK, 2003).

Nesse sentido, as preferências alimentares estão diretamente ligadas ao comportamento de compra dos indivíduos, que, por sua vez, é induzido pela relação direta e indireta com o ambiente micro e macroeconômico.

Este estudo tem como objetivo analisar os hábitos alimentares de famílias de baixa renda de pequenas cidades de MS. Especificamente, pretende-se: i) identificar os hábitos alimentares da população dos territórios CONSAD de MS; ii) analisar as preferências alimentares da população de baixa renda; e iii) identificar segmentos de consumidores.

\section{Material e métodos}

O presente estudo utilizou o banco de dados de um projeto maior, que aborda Segurança Alimentar e Nutricional e o Desenvolvimento Local, conduzido pela UFMS e UCDB. A pesquisa foi realizada junto a 584 famílias, em 24 pequenos municípios de MS, em 2010. Esses municípios compõem os três Consórcios de Segurança Alimentar e Desenvolvimento Local (CONSAD) de MS. Territórios CONSAD são organizações territoriais formalizadas institucionalmente pelo Ministério do Desenvolvimento Social e Combate à Fome (MDS), com um número definido de municípios de baixo IDH e que se agrupam para desenvolver ações, diagnósticos, projetos de SAN e são relacionados a sistemas agroalimentares que visem à luta contra a pobreza e que sejam capazes de interferir na realidade socioterritorial, de forma a integrarem as políticas públicas realizadas nos territórios e envolver os atores sociais (BRASIL, 2008).

O comportamento do consumidor de alimentos foi analisado com base em amostras aleatórias estratificadas de cada território (584 famílias). As entrevistas foram efetuadas por estudantes de graduação e mestrado das duas IES, todos bolsistas do CNPq. A pesquisa abordou os hábitos e preferências alimentares da população de baixa renda (com renda média de até 4 salários mínimos) nesses municípios. Foi utilizado questionário estruturado, denominado "Inquérito de SAN".

Técnicas estatísticas descritivas, como distribuição de frequência, média aritmética e desvio padrão, foram utilizadas na análise quantitativa. Outras técnicas de análise foram: médias, teste Qui-quadrado para as significâncias das médias e análise de agrupamento hierárquica aglomerativa [análise de clusters] (HAIR, JR. et al., 2005). As dimensões que analisadas foram: hábitos (MAIA-FILHO, 2008) e preferências alimentares (LEIBTAG; KAUFMAN, 2003; MURARO, 2007) dos consumidores. As variáveis utilizadas na análise de clusters estão no quadro 1 , inspiradas em Drichoutis, Lazaridis e Nayga (2007).

Quadro 1 - Variáveis utilizadas para a análise de Cluster

\begin{tabular}{|l|}
\hline \multicolumn{1}{|c|}{ Variáveis } \\
\hline Comer Bastante. \\
Comer carne. \\
Várias vezes ao dia em pequenas quantidades. \\
Variado (prato colorido). \\
Várias vezes ao dia em grande quantidade. \\
Comer fruta e verdura. \\
No processo de escolha do alimento a ser consumido, você considera o fato de ele fazer bem à saúde. \\
No processo de escolha do alimento a ser consumido, você considera o preço. \\
No processo de escolha do alimento a ser consumido, você considera o sabor. \\
No processo de escolha do alimento a ser consumidor, você considera o tempo de preparo.
\end{tabular}

Fonte: Elaborado pelos autores. 
O presente estudo não seguiu um modelo específico completo, investigou em pormenores algumas variáveis do modelo de Drichoutis, Lazaridis e Nayga (2007) para definir as escolhas e hábitos alimentares da população de baixa renda dos territórios CONSAD de MS.

\section{Resultados e discussão}

3.1 Hábitos alimentares da população: critério de escolha e preocupação com a saúde

Para avaliar o critério de escolha em relação à compra de alimentos, foi utilizada estatística descritiva, através das variáveis: sabor, preço, se o produto faz bem à saúde e o tempo de preparo. Observando de maneira geral (Gráfico 1), verifica-se que os graus de importância dos atributos analisados são diferentes (teste Qui-quadrado; p-valor < 0,000001).

Gráfico 1 - Atributos mais relevantes na aquisição do produto

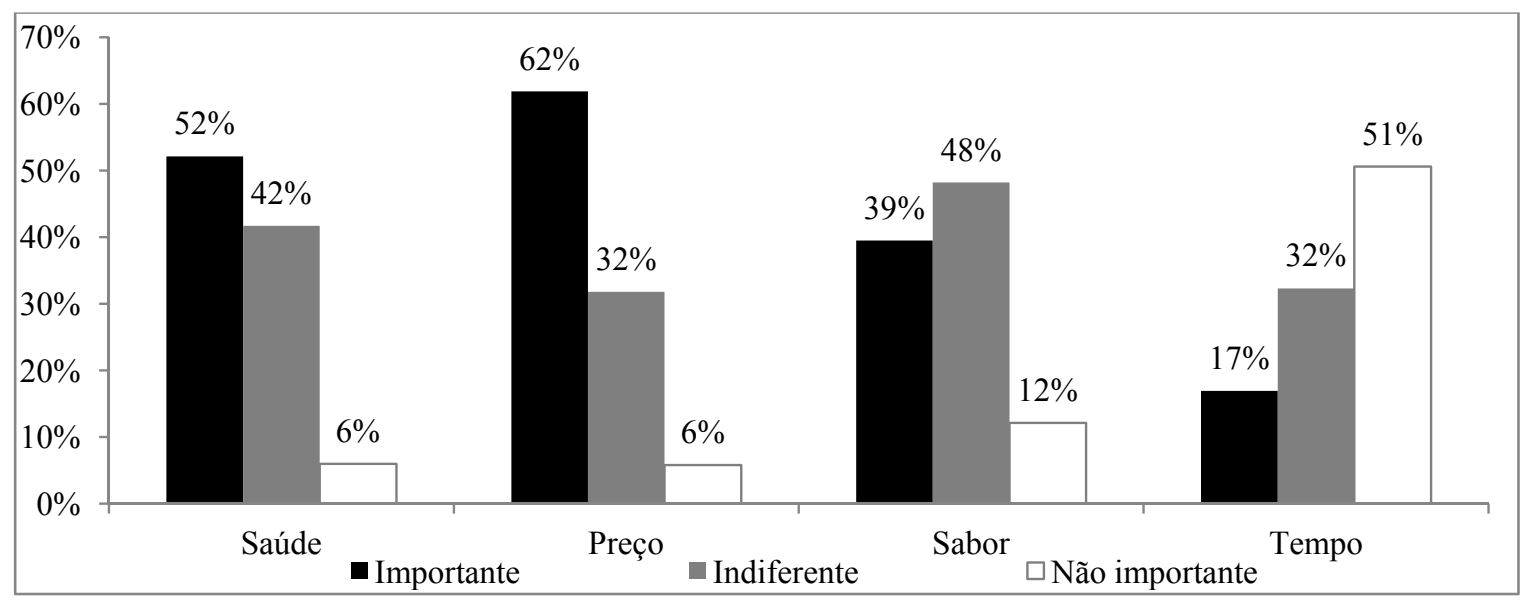

Fonte: Elaborado pelos autores com dados da pesquisa realizada.

Se comparar dois a dois na amostra de 584 pessoas, obter-se-á diferença significativa quando as porcentagens forem maiores que $8 \%$. Dessa forma, pode-se afirmar que, em um nível de confiança de $95 \%$, preço é o atributo mais importante para a populaçãoalvo, seguido da saúde, sabor e tempo. Isso significa que preço, nas famílias de baixa renda, é o principal quesito na hora de escolher um alimento. Estudo realizado por Chauvel e Mattos (2008) confirma esse resultado, no qual os consumidores dedicam muita atenção e tempo às decisões de alocações de recursos (tempo, dinheiro e esforço) e hierarquizam seus gastos em ordem de prioridade, pesquisam preços e buscam organizar suas compras e consumo de forma a evitar desperdícios.

Considerando que possuem recursos escassos para gastarem com alimentos mais caros, e considerando que as formas atuais de distribuição são menos eficientes para famílias de renda baixa, o preço é primordial na escolha dos alimentos nessa classe social. Por outro lado, não significa que a racionalidade econômica seja, de fato, sempre dominante nas escolhas de consumo.

Considerando, ainda, a relação de proporcionalidade inversa entre preço e renda, quanto menor a renda da população, maior a importância do preço como critério de escolha dos produtos alimentícios. Estudo realizado no mesmo território, com uma amostra de 1.200 pessoas, confirma essa afirmação. Oliveira et al. (2010) afirma que variáveis socioeconômicas (renda, escolaridade, tamanho de família) impactam no comportamento de consumo de alimentos, principalmente no que diz respeito ao comportamento saudável; ou seja, quem tem menor renda tende a ser flexível em sua decisão de compra de alimentos quando existem mudanças no ambiente econômico, o que vai de encontro às sugestões da FAO (2008).

Apenas 39\% da população considerou sabor como atributo relevante na escolha dos 
alimentos. Ou seja, os benefícios intrínsecos, como o sabor, não mostram relevância alta entre a população entrevistada, dado que diversas marcas não premium são consumidas de forma a satisfazer as famílias.

Pode-se constatar (Gráfico 2), observando de modo geral, que as considerações sobre a importância dos hábitos alimentares são diferentes ( $p$-valor $<0,000001)$. Se comparar de dois a dois na amostra de 584 pessoas, como pode ser visto na figura 1 , neste também se obterá diferença significativa quando os percentuais forem maiores que $8 \%$. Assim, pode-se afirmar, a um nível de confiança de $95 \%$, que comer frutas e verduras é o mais importante ao se alimentar, seguido de prato variado (colorido), comer carne, comer várias vezes ao dia em pequenas quantidades, comer bastante e, por último, comer várias vezes ao dia em grande quantidade.

Assim, a população de baixa renda mostra-se preocupada com a saúde, e sabe que comer frutas e verduras $(94 \%)$ e comer várias vezes ao dia em pequenas porções (58\%), são práticas adequadas para alimentarse melhor. Porém resta a questão preço, que pode acarretar ainda na escolha errada dos alimentos que fazem bem à saúde.

\subsection{Preferências alimentares da população de baixa renda}

Os dados revelam que a preferência alimentar da população guarda coerência com identidade cultural do Brasil, tratando-se do arroz e feijão como refeição principal, sendo consumidas duas ou mais vezes ao dia; carne de frango, carne suína e peixe foram relatados por mais de $90 \%$ dos entrevistados como alimento indispensável pelo menos uma vez ao dia, assim como frutas $(90,74 \%)$, frituras $(94,50 \%)$, macarrão $(96,26 \%)$ e o ovo $(95,72 \%)$.

Gráfico 2 - Considerações de importância ao se alimentar

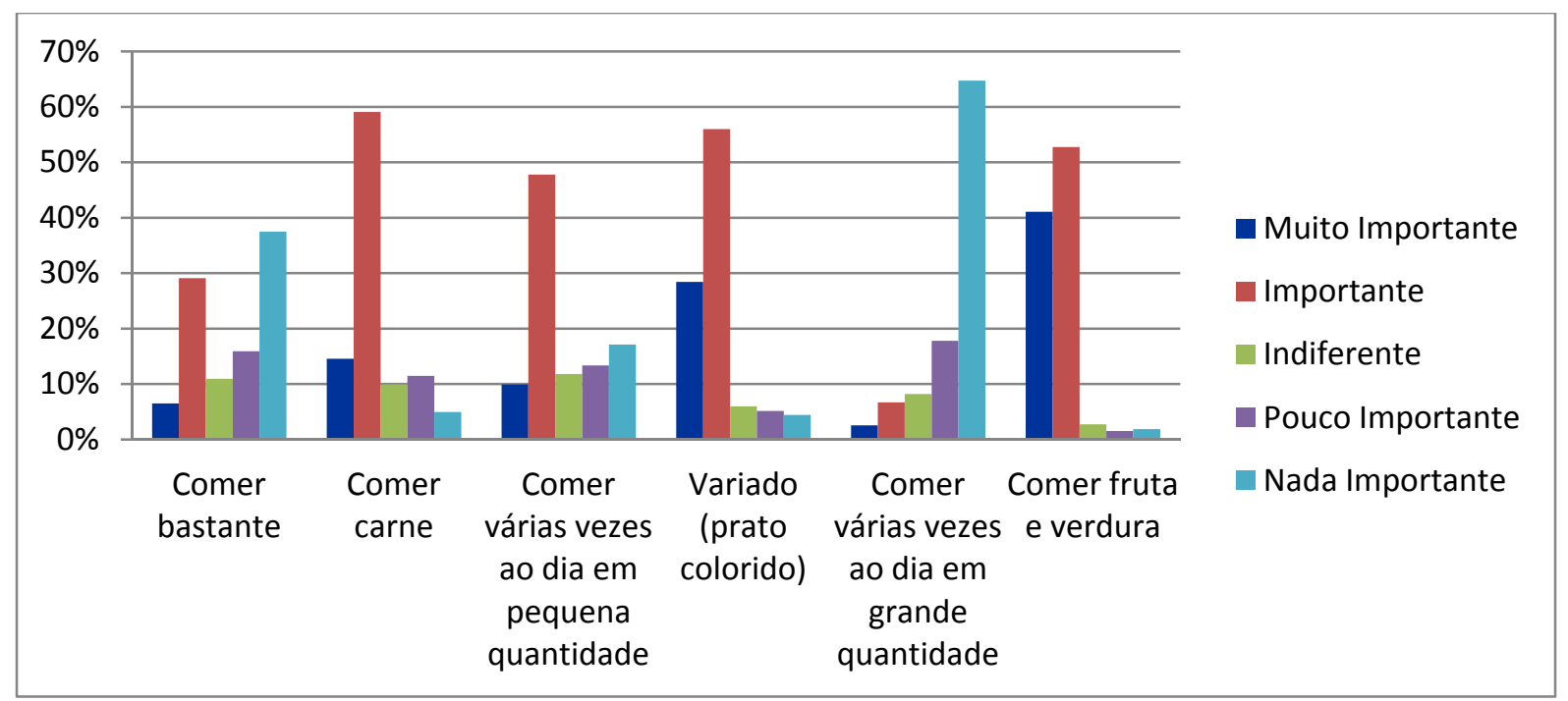

Fonte: Elaborado pelos autores

As verduras e legumes também tiveram grande impacto (mais de $80 \%$ dizem comer pelo menos uma vez ao dia), bem como o leite, relatado por mais de $70 \%$ da população de baixa renda, e carne bovina $(67,81 \%)$. 
Tabela 1 - Preferências alimentares da população de baixa renda

\begin{tabular}{ccc}
\hline \multirow{2}{*}{ Alimentos } & \multicolumn{2}{c}{ Número de vezes no dia } \\
\cline { 2 - 3 } & Frequente (1x/dia) & Muito Frequente (2x ou mais/dia) \\
\cline { 2 - 3 } Arroz & $16,10 \%$ & $83,90 \%$ \\
Feijão & $21,32 \%$ & $78,68 \%$ \\
Carne Bovina & $67,81 \%$ & $32,18 \%$ \\
Carne Suína & $99,03 \%$ & $0,97 \%$ \\
Frango & $96,80 \%$ & $3,20 \%$ \\
Ovo & $95,72 \%$ & $4,28 \%$ \\
Leite & $77,03 \%$ & $22,97 \%$ \\
Peixe & $98,12 \%$ & $1,88 \%$ \\
Verduras / Legumes & $86,07 \%$ & $13,93 \%$ \\
Frutas & $90,74 \%$ & $9,25 \%$ \\
Frituras & $94,50 \%$ & $5,51 \%$ \\
Macarrão & $96,26 \%$ & $3,74 \%$ \\
\hline
\end{tabular}

Fonte: Elaborado pelos autores.

Arroz e feijão é uma combinação de alimentos que não pode faltar na mesa dessa população, sendo os dois alimentos que a população de baixa renda consome com muita frequência todos os dias. Os outros alimentos também são considerados de consumo frequente. O IBGE (2010) confirma que a população de baixa renda é a que mais consome arroz e feijão. Ainda de acordo com o IBGE (2010), corroborando ainda com os resultados encontrados, há um baixo consumo de frutas, legumes e verduras, fonte de vitaminas e minerais, e fibras que são muito importantes para o regulamento intestinal.

\subsection{Cluster de consumidores}

Para verificar a possível existência de segmentos distintos considerando hábitos e preferências alimentares da população de baixa renda de pequenas cidades, foi feita uma análise de clusters utilizando as variáveis do quadro 1. O método escolhido foi o aglomerativo Ward (HAIR et al., 2009), que inicia a análise com um segmento para cada respondente (584) e, a partir daí, vai aglomerando os mais próximos até formar um grande e único segmento, buscando sempre minimizar a variação interna dos agrupamentos.

Para selecionar a quantidade ideal de segmentos, é preciso observar a variação percentual dos coeficientes de aglomeração (Tabela 2). Depois de selecionar a quantidade de segmentos, é necessário identificá-los. Para tanto, foram analisadas as médias de notas atribuídas em cada segmento. Feito o cruzamento com as variáveis elencadas no quadro 1, por meio do teste qui-quadrado, foi avaliada a relação de dependência (Tabela 2).

Tabela 2 - Coeficientes de aglomeração

\begin{tabular}{cccc}
\hline Clusters & Coeficientes de aglomeração & Número de observações & Distância média \\
\hline Cluster 1 & 3485,34 & 353 & 3,017 \\
Cluster 2 & 2193,56 & 231 & 2,97 \\
\hline
\end{tabular}

Fonte: Elaborado pelos autores com fontes de dados primária.

Assim, a análise de clusters identificou dois tipos de segmentos presentes na população de baixa renda, cujo perfil demográfico consta da tabela 3. A maioria dos entrevistados é composta por mulheres (79\%), nos dois segmentos. O elevado percentual de mulheres foi devido ao local e horário de entrevista, que foi realizada de segunda a sábado - nos domicílios, em horário de comercial - o que resultou no fato de que, em grande parte dos casos, os homens se encontravam fora. 
Tabela 3 - Perfil amostra/população

\begin{tabular}{|c|c|c|c|}
\hline Variáveis & \multicolumn{2}{|c|}{ Segmentos } & p-valor \\
\hline Idade & Cluster 1 & Cluster 2 & \\
\hline até 44 & 223 & 165 & 0.039 \\
\hline \multirow[t]{2}{*}{45 ou mais } & 130 & 66 & \\
\hline & \multicolumn{2}{|c|}{ Segmentos } & p-valor \\
\hline $\mathrm{F}$ & 281 & 178 & 0.481 \\
\hline \multirow[t]{2}{*}{ M } & 71 & 52 & \\
\hline & \multicolumn{2}{|c|}{ Segmentos } & p-valor \\
\hline Zona & Cluster 1 & Cluster 2 & \\
\hline Urbano & 297 & 199 & 0.525 \\
\hline \multirow[t]{2}{*}{ Rural } & 54 & 31 & \\
\hline & \multicolumn{2}{|c|}{ Segmentos } & p-valor \\
\hline Escolaridade & Cluster 1 & Cluster 2 & \\
\hline Até ensino primário incompleto & 190 & 132 & 0.39 \\
\hline Ensino primário completo & 37 & 21 & \\
\hline Ensino ginasial incompleto & 57 & 49 & \\
\hline Ensino ginasial completo & 27 & 11 & \\
\hline Ensino médio incompleto & 19 & 14 & \\
\hline Ensino médio completo & 18 & 2 & \\
\hline
\end{tabular}

Fonte: Elaborado pelos autores.

Em sua maioria $(85,2 \%)$, os entrevistados são residentes urbanos. Quanto à escolaridade, $55,3 \%$ tinham ensino primário incompleto - no Brasil este índice é de 44,7\% - de acordo com dados da PNUD (2009). Apenas $3,4 \%$ dessa população entrevistada terminou o ensino médio (contra $24,5 \%$ no Centro-Oeste). A taxa de analfabetismo no Brasil, segundo a PNUD (2009), é de 9,7\% e, no Centro-Oeste, de $8 \%$. Porém a pesquisa demonstra que essa taxa é de 11,01\% nos territórios CONSAD de MS, com índice de escolaridade menor que as médias nacionais e regionais.

A nomeação dos segmentos foi feita de acordo com a frequência das respostas e notas atribuídas às variáveis. $\mathrm{O}$ segmento $X$ foi nomeado de "Saudável" por considerar mais importante o fator saúde na hora de escolher um alimento. E o segmento $Y$ foi designado de "apreciadores de comida" por considerar "comer bastante e sabor" como elementos cruciais na hora da escolha de alimentos.

Ambos os segmentos são marcados por pessoas que consideram importante comer frutas e verduras. Para o segmento "saudável", essa foi uma variável que mais obteve notas altas, sendo considerado determinante na alimentação da população, com nota de
4,34. O p-valor nesses critérios foi significativo. Em sequência, o preço foi destacado com a nota de 3,96 de importância; o prato variado (colorido) ficou com nota de 3,9, também muito importante; comer bastante obteve 1,58 de nota; comer várias vezes ao dia em grandes quantidades ficou com nota 1,37; e comer carne com nota de 1,06, foi destacado como significativo.

O segmento "apreciadores de comida" é formado por pessoas que assumiram a mesma situação de escolha, considerando importante comer frutas e verduras, item com nota de 4,22 . O que se pode considerar aqui é que, mesmo sendo de renda baixa, essa população tem como preocupação alimentar-se de maneira saudável incluindo frutas e verduras na dieta diária. Isso pode ser explicado pelo fato de muitos entrevistados possuírem, em suas casas, hortas para consumo próprio. Aqueles que não produzem para autoconsumo adquirem o produto dependendo do preço e do acesso a esses alimentos. O prato variado (prato colorido), com nota de 4,12 , é muito importante para esse segmento, seguido do preço com 3,78; comer bastante, com 3,93; e sabor, com 3,28 (Tabela 4). 
Tabela 4 - Segmentos dos hábitos e preferências alimentares da população

\begin{tabular}{|c|c|c|c|}
\hline \multicolumn{4}{|c|}{ Notas médias } \\
\hline Variáveis & $\begin{array}{l}\text { Segmento } \\
\text { "saudável" }\end{array}$ & $\begin{array}{c}\text { Segmento } \\
\text { "apreciadores da comida" }\end{array}$ & p-valor \\
\hline Comer bastante & 1,58 & 3,93 & 0,00 \\
\hline Comer carne & 1,06 & 0,88 & 0,00 \\
\hline Várias vezes ao dia em pequenas quantidades & 3,2 & 3,19 & 0,93 \\
\hline Variado (prato colorido) & 3,9 & 4,12 & 0,01 \\
\hline Várias vezes ao dia em grande quantidade & 1,37 & 2,06 & 0,00 \\
\hline Comer fruta e verdura & 4,34 & 4,22 & 0,05 \\
\hline Fazer bem a saúde & 3,7 & 3,58 & 0,12 \\
\hline Preço & 3,96 & 3,78 & 0,03 \\
\hline Sabor & 3,38 & 3,28 & 0,19 \\
\hline Tempo de preparo & 2,4 & 2,32 & 0,44 \\
\hline
\end{tabular}

Fonte: Elaborado pelos autores com dados da pesquisa realizada.

O cluster 1 teve 353 casos $(60,45 \%$ da população) e o cluster 2 teve 231 casos (39,55\% da população). Ao analisar as notas médias obtidas em cada segmento, bem como os seus respectivos p-valores, pode-se perceber seis variáveis que apresentam diferença significativa entre as médias. São elas: comer bastante, comer carne, prato variado, comer várias vezes ao dia em grandes quantidades, comer frutas e verduras e o preço, ou seja, através delas é possível atender de maneira adequada cada um dos segmentos, sendo eles significativos, e confiáveis, pois, o p-valor foi menor que 0,05 . Feito isso, cruzaram-se os segmentos encontrados com os dados sociodemográficos.
O único cruzamento (cross-section) considerado significativo devido ao p-valor menor 0,05 foi a idade versus segmento; então, a idade impacta significativamente no comportamento alimentar da população de baixa renda. Tanto no segmento "saudável" quanto no segmento "apreciadores de comida", a maioria dos entrevistados tem idade menor que 44 anos, com um total de $63,17 \%$ no segmento "saudável" e 71,43\% no segmento "apreciadores de comida" Os demais estão acima dos 45 anos. A análise detalhada da relação entre idade e hábitos e preferências alimentares está representada na tabela 5 .

Tabela 5 - Segmentos dos hábitos e preferências alimentares versus idade da população

\begin{tabular}{|c|c|c|c|c|c|c|c|c|c|c|c|c|}
\hline \multicolumn{13}{|c|}{ Notas médias } \\
\hline \multirow[b]{2}{*}{ Variáveis } & \multicolumn{6}{|c|}{ Segmento X "saudável" } & \multicolumn{6}{|c|}{ Segmento $Y$ "apreciadores da comida" } \\
\hline & Até 18 & $18-24$ & $25-34$ & $35-44$ & $45-54$ & $\begin{array}{c}55 \\
\text { ou mais } \\
\end{array}$ & Até 18 & $18-24$ & $25-34$ & $35-44$ & $45-54$ & $\begin{array}{c}55 \\
\text { ou mais } \\
\end{array}$ \\
\hline Comer bastante & 2,20 & 1,73 & 1,59 & 1,40 & 1,70 & 1,58 & 4,13 & 4,04 & 3,99 & 3,91 & 3,78 & 3,87 \\
\hline Comer carne & 3,20 & 3,43 & 3,49 & 3,47 & 3,54 & 3,50 & 3,75 & 4,08 & 4,07 & 3,75 & 3,89 & 4,00 \\
\hline $\begin{array}{l}\text { Várias vezes ao dia em } \\
\text { pequenas quantidades }\end{array}$ & 2,60 & 3,70 & 3,11 & 3,18 & 3,24 & 3,15 & 3,00 & 3,08 & 3,20 & 3,28 & 3,22 & 3,13 \\
\hline Variado (prato colorido) & 2,80 & 3,60 & 4,04 & 4,07 & 3,84 & 3,73 & 4,00 & 4,08 & 4,03 & 4,21 & 4,22 & 4,13 \\
\hline $\begin{array}{l}\text { Várias vezes ao dia em } \\
\text { grande quantidade }\end{array}$ & 1,80 & 1,73 & 1,43 & 1,28 & 1,39 & 1,20 & 2,88 & 2,13 & 2,03 & 1,98 & 1,94 & 2,17 \\
\hline Comer fruta e verdura & 3,20 & 4,27 & 4,41 & 4,48 & 4,31 & 4,22 & 4,13 & 4,25 & 4,21 & 4,28 & 4,19 & 4,17 \\
\hline Fazer bem a saúde & 3,40 & 3,80 & 3,73 & 3,74 & 3,64 & 3,67 & 3,63 & 3,79 & 3,63 & 3,77 & 3,19 & 3,43 \\
\hline Preço & 3,80 & 3,77 & 4,00 & 3,92 & 4,00 & 3,97 & 3,13 & 3,38 & 3,91 & 3,79 & 3,69 & 3,90 \\
\hline Sabor & 3,00 & 3,33 & 3,44 & 3,33 & 3,40 & 3,40 & 3,75 & 3,46 & 3,38 & 3,33 & 3,00 & 3,03 \\
\hline Tempo de preparo & 2,60 & 2,10 & 2,34 & 2,51 & 2,46 & 2,43 & 2,50 & 2,33 & 2,39 & 2,53 & 2,17 & 1,93 \\
\hline
\end{tabular}

Fonte: elaborado pelos autores com dados da pesquisa realizada 
Observa-se a regularidade nas notas atribuídas aos quesitos, tanto no segmento "saudável", quanto no segmento "apreciadores de comida". Porém o item "comer bastante" é o que apresenta a maior divergência entre os segmentos, visto que, enquanto para o segmento "saudável" ele é considerado de nenhuma a pouca importância, para o segmento "apreciadores de comida", ele é considerado muito importante.

Para o segmento "saudável", um quesito diverge: variedade dos pratos. Nas faixas de idade de 25 a 34 anos e de 35 a 44 anos, o quesito variedade dos pratos é considerado importante, porém, para as demais faixas, é considerado de pouca importância a indiferente. Para a idade inferior a 18 anos, no segmento "saudável", é importante mencionar que a nota atribuída ao quesito "comer fruta e verdura" destoa das demais notas para o mesmo quesito, o que pode ser justificado pelo fato de a população jovem "fugir" de uma alimentação "saudável".

Quando se comparam os dois segmentos, nota-se que o item "comer várias vezes ao dia", é o que apresenta as notas mais baixas dentre os quesitos avaliados, para os dois segmentos, que o consideram um item de nenhuma a pouca importância. Quando se comparam idade versus segmentos, para a faixa etária de 18 a 24 anos, do segmento "saudável", comer bastante é considerado de nenhuma a pouca importância, enquanto na mesma faixa, para o segmento "apreciadores de comida", é considerada importante.

Em consonância aos resultados apresentados, de acordo com Laureatti et al. (2006), as pessoas idosas escolhem alimentos mais saudáveis por se preocuparem com a boa nutrição. Por outro lado, Pagotti (2006) afirma que para os jovens, de modo geral, é considerada uma alimentação equilibrada o estado de bem-estar físico, mental e social, e não simplesmente a ausência de doenças ou debilidades. No entanto muitos jovens têm o FISBERG et al. (2003), conhecimento de uma boa alimentação, mas, em sua maioria, não optam por ela.

\section{Considerações finais}

Esta pesquisa teve como objetivo identificar os hábitos alimentares da população de baixa renda nos territórios CONSAD de MS. Para tanto, foi realizado um survey sobre as preferências e hábitos alimentares da população de baixa renda em 24 pequenas cidades. Buscou-se estudar tais comportamentos a partir de informações reveladas pelos próprios entrevistados sobre os produtos alimentares que mais consomem, as considerações de importância ao se alimentar e os hábitos alimentares.

Com isso, foi possível identificar os alimentos que não podem faltar à mesa do consumidor e os hábitos alimentares. $\mathrm{O}$ principal critério de escolha de alimentos, nessa população, é o preço do alimento. Em segundo lugar, as pessoas são conscientes de que o alimento precisa fazer bem à saúde, mas, muitas vezes, é preciso fazer escolhas que podem não ser as melhores para a saúde devido ao recurso disponível para gastar com alimentação, precisando, em alguns momentos, fazer ponderações sobre o que se pode ou não comprar.

Os alimentos que compõem o prato principal em MS são o arroz e o feijão, combinação consumida pelo menos duas vezes ao dia, assumindo o que foi relatado sobre a identidade cultural do local. Ao se analisar os critérios de comer pelo menos uma vez ao dia, percebe-se que os entrevistados consomem carne de frango, suína, bovina ou de peixe, frutas, verduras, macarrão, fritura, ovo e leite. Eles têm o conhecimento de que, para obter uma alimentação saudável, é essencial comer sistematicamente frutas e hortaliças e ter um prato com diversidade de alimentos (variedade na composição da dieta, como: carboidratos, fibras, legumes e verduras etc.); todavia nem sempre é possível manter essa composição que está relacionada aos recursos disponíveis (dinheiro, esforço, tempo).

Mediante da análise de cluster identificaram-se dois segmentos principais, nomeados pelos autores como "saudáveis", aqueles que têm mais preocupação com a saúde, e "apreciadores de comida", referente ao que gostam de comer bastante e um prato bem colorido, nos quais o preço foi apontado por ambos os segmentos como o critério decisivo na escolha dos alimentos. As duas categorias responderam que o preço é decisivo na hora de comprar os alimentos, sendo o critério de escolha mais importante. Eles também falam 
que o prato precisa ser variado, comer em pequenas porções várias vezes ao dia e que o alimento precisa fazer bem à saúde.

A idade da população tem importância significativa na escolha dos alimentos. De um lado, o mais jovem, mesmo conhecendo alguns conceitos sobre a alimentação saudável, nem sempre opta por ela; por outro lado, os idosos preferem a redução em comidas muito gordurosas e começam a ingerir menos açúcares e diminuem o nível de sal.

Os dados deste estudo podem ser úteis tanto para as políticas públicas quanto para a classe empresarial. No que diz respeito a políticas públicas, o conhecimento das escolhas alimentares ser dependente do recurso disponível (dinheiro, tempo e esforço) para essa população poderia contribuir para uma melhor orientação das políticas de distribuição de renda e saúde pública, que pretendem melhorá-las através da alimentação. Apresenta dois segmentos de consumidores dentro dessa população, podendo contribuir para a orientação e a realização de campanhas de reeducação alimentar para populações de baixa renda, na busca de informações sobre a maneira que o indivíduo pode usar os alimentos disponíveis para sua alimentação. $\mathrm{Ou}$ seja, além de proporcionar uma melhor distribuição de alimentos e com preços menores, as políticas públicas devem colocar em sua envergadura programas que conscientizem a população sobre nutrição.

No que diz respeito a contribuições gerenciais, este estudo oferece a caracterização do comportamento de consumidor de alimentos de baixa renda, residentes em pequenas cidades, que serve como base para desenvolvimento de estratégias orientadas para o mercado, conhecimento essencial para que os agentes da cadeia produtiva (agricultores, fabricantes e varejistas) agreguem valor aos produtos, podendo direcionar tipos de alimentos para as diferentes classes sociais. À luz da academia, o estudo contribui com a caracterização do comportamento do consumidor de alimentos no que diz respeito à escolha e aos hábitos e preferências alimentares.

\section{Referências}

BELIK, W. Perspectiva para a segurança alimentar e nutricional no Brasil. Saúde e sociedade, São Paulo, v. 12, n. 1, p. 12-20, 2003.

BRADY, M. K.; ROBERTSON, C. J.; CRONIN, J. J. Managing behavioral intentions in diverse cultural environments: an investigation of service quality, service value, and satisfaction for American and Ecuadorian fast-food customers. Journal of International Management, v. 7, n. 2, p. 129-149, 2001.

BRASIL. Ministério do Desenvolvimento Social e Combate à Fome (MDS), 2008. Disponível em: <http:/ / www.mds. gov.br/programas/seguranca-alimentar-e-nutricionalsan/consad>. Acesso em: 08 fev. 2009.

CANESQUI, A. M.; GARCIA, R. W. D. Uma introdução à reflexão sobre a abordagem sociocultural da alimentação. In: CANESQUI, A. M.; GARCIA, R. W. D. (Org.). Antropologia e nutrição: um diálogo possível. Rio de Janeiro: Fiocruz, 2005.306p. (Col. Antropologia e Saúde).

CHAUVEL, M. A.; MATTOS, M. P. A. Z. Consumidores de baixa renda: uma revisão dos achados de estudos feitos no Brasil. Cadernos EBAPE.BR. Rio de Janeiro, v. 6, n. 2, p. 1-17, jun. 2008.

CONTRERAS, J.; GRACIA, M. Alimentação, sociedade e cultura. Rio de Janeiro: Fiocruz, 2011. 496p.

CUDJE, G.; BREISINGER, C.; DIAO, X. Local impacts of a global crisis: food price transmission, consumer, welfare and poverty in Ghana Food Policy. In press, available online, 2010. Disponível em: <http:/ /www. ifpri.org/sites/default/files/publications/gsspwp15. pdf $>$. Acesso em: 20 out. 2013.

DRICHOUTIS, A. C.; LAZARIDIS, P.; NAYGA JR, R. M. An assessment of product classe involvement in food purchasing behavior. European Journal of Marketing, v. 41, n. 7-8, 2007, p. 888-914.

FISBERG, M. et al. Hábitos alimentares na adolescência. Pediatr Mod, v. 36, p. 766-70, 2000. In: GARCIA, R. W. D. Reflexos da globalização na cultura alimentar: considerações sobre as mudanças na alimentação urbana. Revista de Nutrição, Campinas, p. 483-492, out./ dez. 2003.

FOOD AND AGRICULTURAL ORGANIZATION OF THE UNITED NATIONS - FAO. The state of food insecurity in the world 2008: high food price and food security - threats and opportunities, 2008. Disponível em: <http://www.fao.org/docrep/011/i0291e/i0291e00. htm>. Acesso em: 24 out. 2013.

HAIR, J. F. et al. Análise multivariada de dados. 6. ed. Porto Alegre: Bookman, 2009.

INGLIS, V.; BALL, K.; CRAWFORD, D. Does modifying the household food budget predict changes in the healthfulness of purchasing choices among low- and high-income women? Appetite, v. 52, n. 2, p. 273-279, abr. 2009.

INSTITUTO BRASILEIRO DE GEOGRAFIA E ESTATÍSTICA - IBGE. Censo Agropecuário 2006: Agricultura Familiar. Rio de Janeiro, 2006, p.1-267.

. Pesquisa de orçamentos familiares 2008-2009: despesas, rendimentos e condições de vida. Rio de Janeiro, 2010. 
LAURETTI, M. et al. Sensory acceptability of traditional food preparations by elderly people. Food quality and preference, v. 17, n. 1-2, p. 43-52, jan./mar. 2006.

LEIBTAG, E. S; KAUFMAN, P. R. Exploring food purchase behavior of low-income households: how do they economize? Agriculture Information Bulletin, n. 747, 2003. Disponível em: <http://www.ers.usda.gov/Publications/AIB747/aib74707.pdf>. Acesso em: 18 abr. 2009.

LENGARD, V.; JOHANSEN, S. B.; HERSLETH, M. Alternative methods for combining design variables and consumer preference with information about attitudes and demographics in conjoint analysis. Food Quality and Preferences, v. 21, n. 4, p. 368-378, jun. 2010.

LIMA-FILHO, D. O.; OLIVEIRA, L. D. S. Food distribution retail technologies: a comparison between countries with different income levels. Espacios, Caracas, v. 30, n. 3, 2009, p. 21-24.

MAIA-FILHO, N. S. Análise descritiva dos hábitos de lazer do consumidor idoso de baixa renda. 2008. 140 f. Dissertação (Mestrado em Administração) - Universidade Federal do Ceará, Fortaleza, 2008.

MISHRA, G., BALL, K., ARBUCKLE, J., CRAWFORD, D. Dietary patterns of Australian adults and their association with socioeconomic status: results from the 1995 National Nutrition Survey. Eur J Clin Nutr, v. 56, n. 7, 2002, p. 687-93.

MONTEIRO, C. A. A dimensão da pobreza, da desnutrição e da fome no Brasil. Revista Estudos Avançados, São Paulo, v. 17, n. 48, p. 7-20, 2003.

MYERS, R. J. On the cost of food price fluctuations in low-income countries. Food Policy, v. 31, n. 4, 2006, p. 288-301.
OLIVEIRA, L. D. S.; WATANABE, E. A. M.; LIMA-FILHO, D. L.; SPROESSER, R. L. Public policies for food security in countries with diferents income levels. International Public Management Review, v. 11, n. 3, p. 122-141, 2010.

ORGANIZAÇÃO MUNDIAL DA SAÚDE - OMS. Diet, nutrition and the prevention of excess weight gain and obesity. Geneva: WHO, 2003. Disponível em: <http:// whqlibdoc.who.int/trs/WHO_trs_916.pdf> Acesso em: 17 out. 2009.

PAGOTTI, A. W. et al. As preocupações dos estudantes universitários do curso de Psicologia de duas instituições de ensino superior, 2006. Disponível em: <http://www. psicologia.com.pt/artigos/textos/A0324.pdf>. Acesso em: 20 jul. 2010.

PROGRAMA DAS NAÇÕES UNIDADES PARA O DESENVOLVIMENTO - PNUD, 2009. Disponível em: <http://www.pnud.org.br/pobreza_desigualdade/ reportagens $/$ index.php?id01=3187\&lay $=$ pde $>$. Acesso em: 27 out. 2013.

POULAIN, J-P. Sociologias da alimentação. Florianópolis: Editora UFSC, 2004.

POWELL, L. M.; ZHAO, Z.; WANG, Y. Food prices and fruit and vegetable consumption among young American adults. Health $\mathcal{E}$ Place, v. 15, n. 4, p. 1064-1070, 2009.

REGMI, A.; GEHLHAR, M. New directions in global food markets. Agriculture Information Belletin Number, n. 794, p. 1-81, 2005.

WEINGARTNER, L. The concept of food and nutrition security. Background Paper n. 1 in International Training Course. Food and nutrition security: Assessment instruments and intervention strategies. Welt Hunger Hilfe, 2004. 